Research article

Open Access

\title{
Systemic chemotherapy induces microsatellite instability in the peripheral blood mononuclear cells of breast cancer patients
}

\author{
Fernando LA Fonseca ${ }^{1,2}$, Aleksandra VL Sant Ana ${ }^{1,2}$, Israel Bendit ${ }^{3}$, Vitor Arias ${ }^{4}$, Luciano J Costa ${ }^{5}$, \\ Aparecida A Pinhal 6 and Auro del Giglio ${ }^{1,2}$
}

\author{
${ }^{1}$ ABC Foundation School of Medicine, Hematology and Oncology, Santo Andre, São Paulo, Brazil \\ 2Faculdade de Medicina da Universidade de São Paulo, Hematology Post Graduate Section, São Paulo, Brazil \\ 3Hemocentro de São Paulo, Tumor Biology, São Paulo, Brazil \\ ${ }^{4}$ Instituto Adolfo Lutz, Anatomic Pathology, São Paulo, Brazil \\ ${ }^{5}$ Faculdade de Medicina da Universidade de São Paulo, Pathology Post Graduate Section, São Paulo, Brazil \\ ${ }^{6} \mathrm{ABC}$ Foundation School of Medicine, Biochemistry, São Paulo, Brazil
}

Corresponding author: Auro del Giglio, sandrabr@netpoint.com.br

Received: 13 May 2004 Revisions requested: 15 Jul 2004 Revisions received: 15 Sep 2004 Accepted: 24 Sep 2004 Published: 04 Nov 2004

Breast Cancer Res 2005, 7:R28-R32 (DOI 10.1186/bcr950)

(C) 2004 Fonseca et al., licensee BioMed Central Ltd.

This is an Open Access article distributed under the terms of the Creative Commons Attribution License (http://creativecommons.org/licenses/by/ 2.0), which permits unrestricted use, distribution, and reproduction in any medium, provided the original work is cited.

\begin{abstract}
Introduction Systemic chemotherapy is an important part of treatment for breast cancer. We conducted the present study to evaluate whether systemic chemotherapy could produce microsatellite instability (MSI) in the peripheral blood mononuclear cell fraction of breast cancer patients.

Methods We studied 119 sequential blood samples from 30 previously untreated breast cancer patients before, during and after chemotherapy. For comparison, we also evaluated 20 women who had no relevant medical history (control group).

Results In 27 out of 30 patients we observed MSI in at least one sample, and six patients had loss of heterozygosity. We found a significant correlation between the number of MSI events per

sample and chemotherapy with alkylating agents $(P<0.0001)$. We also observed an inverse correlation between the percentage of cells positive for hMSH2 and the number of MSI events per sample $(P=0.00019)$ and use of alkylating agents $(P=0.019)$.

Conclusion We conclude that systemic chemotherapy may induce $\mathrm{MSI}$ and loss of heterozygosity in peripheral blood mononuclear cells from breast cancer patients receiving alkylating agents, possibly mediated by a chemotherapyinduced decrease in the expression of hMSH2. These effects may be related to the generation of secondary leukaemia in some patients, and may also intensify the genetic instability of tumours and increase resistance to treatment.
\end{abstract}

Keywords: cyclophosphamide, microsatellite repeats, neoplasms, proliferating cell nuclear antigen, second primary

\section{Introduction}

Systemic chemotherapy is an important part of treatment for breast cancer in both the adjuvant and palliative settings. Despite the consistent improvement in overall survival afforded by systemic adjuvant chemotherapy, about $1 \%$ of patients develop secondary leukaemia and/or myelodysplasia, probably as a consequence of the genotoxic effects of this type of treatment [1]. Deficiencies in the DNA mismatch repair system leading to microsatellite instability (MSI) may produce a syndrome of familial predisposition to colon, endometrial and upper gastrointestinal cancers, known as hereditary nonpolyposis colon cancer [2]. The
DNA mismatch repair system depends on the coordinated interplay of several proteins encoded by various genes, including hMLH1, hMSH2, hPMS1 and hPMS2 [2]. Several groups have reported a significant association between MSI and treatment-related secondary acute myeloid leukaemia (AML) and myelodysplasia [3-5]. In some studies MSI in treatment-related secondary AML/myelodysplasia cases was accompanied by hMLH1 hypermethylation [3] and $\mathrm{MSH} 2$ polymorphisms [6].

In order to evaluate whether systemic chemotherapy could produce MSI in normal peripheral blood mononuclear cells

$\overline{\mathrm{AML}}=$ acute myeloid leukaemia; $\mathrm{LOH}=$ loss of heterozygosity; $\mathrm{MSI}=$ microsatellite instability; PBMC = peripheral blood mononuclear cell; PCNA $=$ proliferating nuclear antigen; $\mathrm{PCR}=$ polymerase chain reaction. 
(PBMCs), we analyzed PBMCs from breast cancer patients collected before, during and after systemic chemotherapy.

\section{Methods}

\section{Collection and preparation of samples}

This protocol was approved by our institutional review board. We obtained blood samples from 33 patients with histologically confirmed breast cancer after informed consent had been obtained. We had only the initial sample in three patients, and so we could not include them in the study. We therefore studied 119 sequential blood samples from 30 previously untreated breast cancer patients, collected at 3-month intervals before, during and after receiving systemic treatment (13 adjuvant, 12 neoadjuvant and 5 palliative). Three patients initially received hormones (two adjuvant and one palliative).

Chemotherapy combinations containing cyclophosphamide were classified as alkylating regimens (FAC [5-fluorouracil, doxorubicin and cyclophosphamide], AC [doxorubicin and cyclophosphamide], CMF [cyclophosphamide, methotrexate, and 5-fluorouracil]). We also studied PBMCs from 20 normal control women, who had no relevant previous medical history, by immunocytochemistry. Afterward, we collected peripheral blood from these 20 normal control women on two occasions with a 3-month interval to evaluate MSI using the TP53Alu and PCR15.1 markers (described below).

Each sample consisted of $20 \mathrm{ml}$ venous blood, from which we separated the PBMCs by Ficoll gradient (Ficoll Hypaque; Organon Teknica ${ }^{\circledR}$, Durham, NC, USA), yielding a final concentration of $1.0 \times 10^{6} \mathrm{cells} / \mathrm{ml}$; we sent part of the sample for cytospin for immunocytochemical studies and part for DNA extraction. DNA was extracted from mononuclear fraction by the use of Trizol (Invitrogen, Carlsbad, CA, USA), in accordance with the manufacturer's instructions. For immunocytochemistry analysis, assay slides were prepared (described in detail elsewhere [7]).

\section{Microsatellite analysis}

The sequences of all primers used for six microsatellite loci (BAT-26, BAT-40, MFD-28, MFD-41, TP53, PCR15.1, TP53ALU) and the PCR protocols used to study each of these markers were described previously [8]. Briefly, PCR products were denatured and subjected to electrophoresis in Gene Gel Clean 15/24 (Amershan Pharmacia Biotech $\mathrm{AB}$, Uppsala, Sweden) for $90 \mathrm{~min}$ at $600 \mathrm{~V}$ and $8^{\circ} \mathrm{C}$, and then silver stained using a Hoefer Automed Gel Stainer (Amershan Pharmacia Biotech AB). MSI was defined by the appearance of novel bands and loss of heterozygosity (LOH) whenever disappearance of previously visualized bands occurred, as described previously [8]. Three inde-
Table 1

Peripheral blood mononuclear cells positive for each of the studied markers by immunocytochemistry

\begin{tabular}{ll}
\hline Protein & Percentage \\
\hline PCNA & $15.93 \pm 4.38$ \\
hMLH1 & $0.25 \pm 1.11$ \\
hPMS1 & $0.6 \pm 0.99$ \\
hPMS2 & $0 \pm 0$ \\
hMSH2 & $72.7 \pm 20.33$ \\
TP53 & $0 \pm 0$ \\
\hline
\end{tabular}

Values are expressed as mean \pm standard deviation (\%).

pendent observers analyzed the results of each gel for $\mathrm{LOH}$ and MSI.

\section{Immunocytochemistry}

For immunostaining for proliferating nuclear antigen (PCNA), hMLH1, hPMS1, hPMS2, hMSH2 and TP-53 proteins, we used an avidin-biotin-peroxidase complex and 3,3'-diaminobenzidine as chromogen. All antibodies were supplied by Santa Cruz Biotechnology (Santa Cruz, CA, USA), and for each clone we employed the following dilutions: PC-10 (anti-PCNA), 1:1000; C-20 (anti-hMLH1), 1:25; K-20 (anti-hPMS1), 1:25; C-20 (anti-hPMS2), 1:200; N-20 (anti-hMSH2), 1:200; and Pab1801 (antiP53), 1:400. Endogenous peroxidase activity was blocked by incubation with $\mathrm{H}_{2} \mathrm{O}_{2}$ and washed in tap water. The cytospin slides were then treated with $7 \%$ skimmed milk for 60 min to block nonspecific protein binding. Each antibody was applied separately, and cytospins were incubated for 18 hours at $3^{\circ} \mathrm{C}$. After 3 washes in phosphate-buffered saline, antigen-bound primary antibody was detected using a standard streptavidin-biotin complex (Strep AB Complex; Dako, Carpinteria, CA, USA). After brief washing, slides were incubated with diaminobenzidine and $\mathrm{H}_{2} \mathrm{O}_{2}$ for $10 \mathrm{~min}$ and then counterstained with haematoxylin, dehydrated in graded alcohols and cleared in xylene. Two independent observers scored 300 cells/slide as positive or negative according to the presence of nuclear staining for each of the aforementioned antibodies. Then, the results from these two observers were averaged to obtain a percentage of positive cells per sample.

\section{Statistical methods}

We analyzed correlations between categorical variables using the $\chi^{2}$ or Fisher's exact test. We used analysis of variance to study correlations between continuous and categorical variables, and simple regression to analyze correlations between continuous variables.

\section{Results}

We studied 30 patients with median age 52 years (range 25-80 years). Twelve patients had stage II, 13 had stage III 
Figure 1

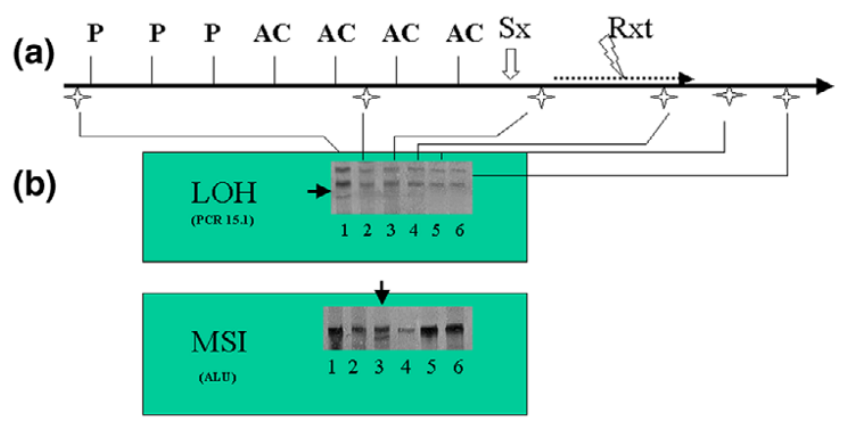

Loss of heterozygosity (LOH) and microsatellite instability (MSI) occurrence. (a) The clinical course of one of the studied patients who received three cycles of neoadjuvant paclitaxel $(P)$ followed by four cycles of neoadjuvant doxorubicin with cyclophosphamide (AC) before surgery (Sx) and radiation therapy (Rxt). Each star below the straight line indicates a blood sample collection (time intervals between collections are not to scale). (b) Single-strand conformation polymorphism (SSCP) gels for two MSI markers PCR15.1 and ALU from the patient represented in panel a, showing the occurrence of $\mathrm{LOH}$ and MSI (black arrows). Each lane corresponds to one of the blood samples collected from this patient. Note that MSI disappears whereas LOH persists.

and five had stage IV breast cancer. We evaluated samples from 20 normal control women by immunocytochemistry for PCNA, hMLH1, hPMS2, hMSH2 and TP-53 (Table 1); the raw data for each of the included patients appear in Additional file 1.

We observed MSI in 40 out of the 119 samples collected, and MSI was observed in 27 out of 30 patients in at least one sample. We observed LOH at the PCR15.1 locus in six patients. Whereas MSI was usually transient, disappearing in the next collected sample, $\mathrm{LOH}$ was persistent in all subsequent samples in five of the six observed cases (Fig. 1).

In order to ensure that our findings in the patients studied were not due to chance, we studied 20 normal blood donors (women who had no relevant medical history; see Additional file 2). In those women blood samples were collected twice, with a 3-month interval between samples. We studied both samples from each of these normal women for MSI using TP53ALU and PCR15.1 markers. In only one normal woman was any MSI identified (one additional band was detected in the PCR15-1 marker after 3 months). None of them had LOH (data not shown). Therefore, in only one blood sample out of 40 drawn from these 20 normal women was there any evidence of $\mathrm{MSI}$, as compared with 28 out of 119 samples from the breast cancer patients studied using the same markers $(P=0.018)$.

We observed a significant correlation between the number of $\mathrm{MSI}$ events per sample and the use of chemotherapy $(P$ $=0.005)$, especially chemotherapy containing alkylating agents $(P<0.0001$; Fig. 2$)$. We also observed an inverse correlation between the number of $\mathrm{MSI}$ events per sample and the percentage of cells positive for MSH2 by immunocytochemistry $(P=0.00019)$ and especially for the MFD41 marker $(P=0.000638)$. The percentage of cells positive for $\mathrm{MSH} 2$ by immunocytochemistry was inversely correlated with the use of chemotherapy regimens containing alkylating agents $(P=0.019)$. We found no significant correlations between the number of $\mathrm{MSI}$ events or the presence of $\mathrm{LOH}$ and the frequency of cells positive for TP53, hMLH1, hPMS1 and hPMS2.

In the present study, the percentage of cells positive for PCNA, as determined by immunocytochemistry, was directly correlated with use of radiation therapy $(P=0.046)$ and with the percentage of MSH2-positive cells $(P=$ $0.000096)$, and inversely correlated with the presence of $\mathrm{LOH}(P=0.043)$, but it was not correlated with the number of MSI events per sample.

\section{Discussion}

Systemic chemotherapy administered to breast cancer patients induces secondary leukaemia and myelodysplasia in about $1 \%$ [1]. In our study, in $90 \%$ of patients MSI was noted in at least one of the samples. This finding does not appear due to chance because only one of the sequential blood samples drawn from 20 normal donor women exhibited MSI. Furthermore, we observed a significant and direct correlation between the number of $\mathrm{MSI}$ events per sample and the use of chemotherapy, especially with regimens containing alkylating agents. We believe that our data reflect the genotoxic effects of chemotherapy on normal cells because in only one normal control woman out of 20 was there evidence of MSI (after 3 months) and with only one of the two surveyed MSI markers. Furthermore, no $\mathrm{LOH}$ was observed within this cohort of normal women. Therefore, because MSI and LOH occurred at significantly greater frequency among patients with breast cancer, it is unlikely that our findings could be due to normal variations in $\mathrm{MSI}$ occurrence or to technical artifacts.

We also noted a significant, inverse correlation between the number of MSI events per sample and the expression of hMSH2 by immunocytochemistry. Because we noted fluctuation in hMSH2 levels throughout the treatment of several patients, it is possible that $\mathrm{hMSH} 2$ downregulation occurs at the post-transcriptional level and could be related to the use of chemotherapy based on alkylating agents. Interestingly, Watanabe and coworkers [9] described induction of $\mathrm{MSI}$ in ovarian tumours from patients studied before and after they received cisplatin-based chemotherapy, but in their study the cisplatin-induced MSI correlated with a significant decrease in hMLH1 expression. 

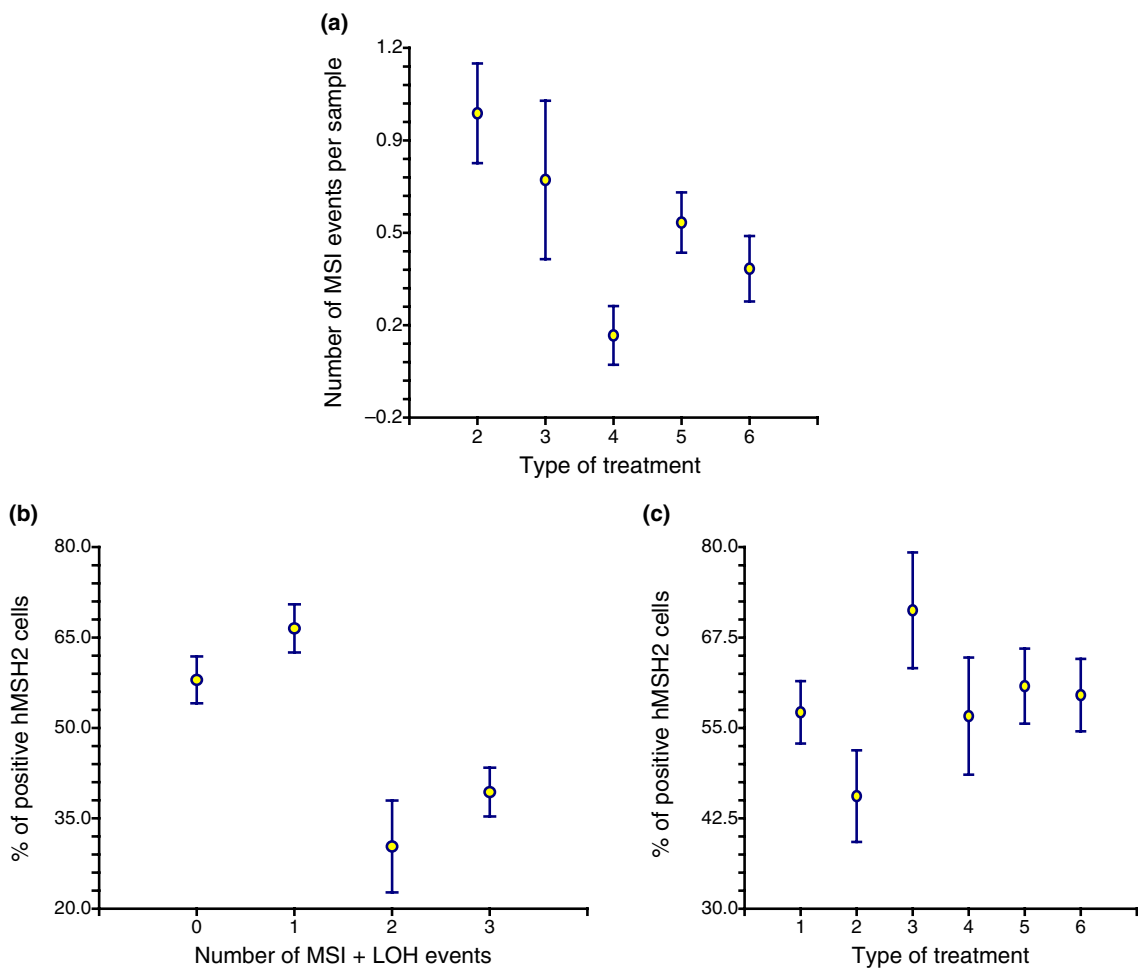

Correlations between microsatellite instability (MSI) events, treatment, and hMSH2-positive cells. Significant correlations between (a) number of $\mathrm{MSI}$ events and type of treatment received by patients during which samples were collected (2, alkylating chemotherapy; 3 , nonalkylating chemotherapy; 4, radiation therapy; 5 , hormone therapy; and 6, off treatment), (b) percentage of hMSH2-positive cells and number of MSI events (MSI and loss of heterozygosity [ $\mathrm{LOH}]$ ), and (c) percentage of hMSH2-positive cells and type of treatment received by patients during which samples were collected (1, before treatment; 2 , alkylating chemotherapy; 3 , nonalkylating chemotherapy; 4 , radiation therapy; 5 , hormone therapy; 6 , off treatment). Values are expressed as means and standard errors (for $P$ values see text).

We believe that the transient decrease in $\mathrm{hMSH} 2$ expression that we noted might have predisposed to an increased number of MSI events. In fact, Zhu and coworkers [10] also described abnormal hMSH-2 expression in about one-third of cases of AML, mainly in those with treatment-related secondary $A M L$ and in patients who were elderly.

PCNA is a $36 \mathrm{kDa}$ nuclear protein that functions as a cofactor for $\delta$-DNA polymerase, which is regulated in a cell cycle dependent manner. PCNA expression also increases when cells are actively engaged in DNA repair [11]. The correlation of PCNA with $\mathrm{LOH}$ but not with the number of MSI events found in the present study may indicate that PCNA may have greater expression in situations of more intense DNA damage that tend to be long-lasting. Interestingly, our group previously showed that PCNA over-expression was associated with resistance to chemotherapy AML and chronic lymphocytic leukaemia [12,13].

\section{Conclusion}

We conclude that systemic chemotherapy may induce MSI and $\mathrm{LOH}$ in PBMCs from breast cancer patients receiving chemotherapy regimens, especially chemotherapy regi- mens containing alkylating agents. Furthermore, it is possible that these alterations are associated with a chemotherapy-induced decrease in the expression of $\mathrm{hMSH}$ 2. These effects may be related to the generation of secondary leukaemia in some patients, and may also intensify the genetic instability of tumours and increase resistance to treatment.

\section{Competing interests}

The author(s) declare that they have no competing interests.

\section{Additional files}

The following Additional files are available online:

\section{Additional File 1}

An Excel file showing raw data for each patient studied. See http://www.biomedcentral.com/content/ supplementary/bcr950-S1.xls 


\section{Additional File 2}

A figure showing the results of testing in 20 normal women. The 20 normal women were tested for MSI using the PCR15.1 and TP53Alu markers. Each normal woman's sample corresponds to one letter (e.g. 'A' represents the first sample of the first control woman and 'A1' her second sample, collected 3 months latter). We noted only one case (S and S1) in which the second sample showed an extra band with the marker PCR15.1 (indicated by an arrow).

See http://www.biomedcentral.com/content/

supplementary/bcr950-S2.ppt

\section{Acknowledgement}

This study was supported by grant FAPESP $N^{\circ} 00 / 04681-2$. This work was previously presented as a poster at the 2004 ASCO meeting in New Orleans.

\section{References}

1. Bernard-Marty C, Mano M, Paesmans M, Accettura C, Munoz-Bermeo R, Richard T, Kleiber K, Cardoso F, Lobelle JP, Larsimont D, et al:: Second malignancies following adjuvant chemotherapy: 6-year results from a Belgian randomized study comparing cyclophosphamide, methotrexate and 5-fluorouracil (CMF) with an anthracycline-based regimen in adjuvant treatment of node-positive breast cancer patients. Ann Oncol 2003, 14:693-698.

2. Chung DC, Rustgi AK: The hereditary nonpolyposis colorectal cancer syndrome: genetics and clinical implications. Ann Intern Med 2003, 138:560-570.

3. Das-Gupta EP, Seedhouse $\mathrm{CH}$, Russell NH: Microsatellite instability occurs in defined subsets of patients with acute myeloblastic leukaemia. Br J Haematol 2001, 114:307-312.

4. Sheikhha MH, Tobal K, Liu Yin JA: High level of microsatellite instability but not hypermethylation of mismatch repair genes in therapy-related and secondary acute myeloid leukaemia and myelodysplastic syndrome. Br J Haematol 2002, 117:359-365.

5. Olipitz W, Hopfinger G, Aguiar RC, Gunsilius E, Girschikofsky M, Bodner C, Hiden K, Linkesch W, Hoefler G, Sill H: Defective DNA-mismatch repair: a potential mediator of leukemogenic susceptibility in therapy-related myelodysplasia and leukemia. Genes Chromosomes Cancer 2002, 34:243-248.

6. Worrillow LJ, Travis LB, Smith AG, Rollinson S, Smith AJ, Wild CP, Holowaty EJ, Kohler BA, Wiklund T, Pukkala E, et al:: An intron splice acceptor polymorphism in hMSH2 and risk of leukemia after treatment with chemotherapeutic alkylating agents. Clin Cancer Res 2003, 9:3012-3020.

7. Borgen E, Naume B, Nesland JM, Kvalheim G, Beiske K, Fodstat O, Diel I, Solomayer E-F, Theocharous P, Coombes RC, et al.: Standardization of the immunocytochemical detection of cancer cells in BM and blood: I. Estabilishment of objective criteria for the evaluation of immunostained cells. Cytotherapy 1999, 1:377-388

8. Dietmaier W, Wallinger S, Bocker T, Kullmann F, Fishel R, Ruschoff J: Diagnostic microsatellite instability: definition and correlation with mismatch repair protein expression. Cancer Res 1997, 57:4749-4756.

9. Watanabe $\mathrm{Y}$, Koi M, Hemmi H, Hoshai $\mathrm{H}$, Noda $\mathrm{K}$ : A change in microsatellite instability caused by cisplatin-based chemotherapy of ovarian cancer. Br J Cancer 2001, 85:1064-1069.

10. Zhu YM, Das-Gupta EP, Russell NH: Microsatellite instability and p53 mutations are associated with abnormal expression of the MSH2 gene in adult acute leukemia. Blood 1999, 94:733-740.

11. Paunesku T, Mittal S, Protic M, Oryhon J, Korolev SV, Joachimiak A, Woloschak GE: Proliferating cell nuclear antigen (PCNA): ringmaster of the genome. Int J Radiat Biol 2001, 77:1007-1021.

12. Del Giglio A, Drach J, Kornblau SM, Patel S, Novaes M, Khetan R, Sawaya N, Dorlhiac-Llacer P, Chamone DF, Andreeff M: Quantitative expression of proliferating cell nuclear antigen by western blot (PCNAWB) in peripheral blasts correlates with remission induction in patients with acute myelogenous leukemia. Leuk Lymphoma 1995, 19:235-241.

13. Del Giglio A, O'Brien S, Ford RJ Jr, Manning J, Saya H, Keating M, Johnston D, Chamone DF, Deisseroth AB: Proliferating cell nuclear antigen (PCNA) expression in chronic lymphocytic leukemia (CLL). Leuk Lymphoma 1993, 10:265-271. 